\title{
Interactive comment on "The efficacy of aerosol-cloud-radiative perturbations from near-surface emissions in deep open-cell stratocumulus" by Anna Possner et al.
}

\section{Anonymous Referee \#1}

Received and published: 11 September 2018

Possner et al. present a modelling study in which the effects of idealised ship emissions on clouds and radiation are examined. Tools and analysis are adequate, and the diligent analysis by Possner et al. allowed for very important insights. A particularly important finding is that a substantial increases in all-sky albedo even relatively far away from the aerosol emission source may be expected, and that there is no simple way of identifying these changes given the large natural variability of the cloud properties. With a known location of the emission source, in turn, detection and attribution seems feasible.

The study is very relevant to the readership of Atmos. Chem. Phys. and should be 
published after some minor revisions.

The most important one is that the authors need to clarify better than they did which quantities are reported as all-sky, and which as cloudy-sky averages. Some further mistakes or unclear aspects that I list below also should be corrected.

\section{Specific comments}

p1119 - the forcing is for the anthropogenic perturbation of the aerosol

p2I5 - invert the order of the Durkee references

p2I15 - Toll rather did conclude that the LWP change was small

p2l19 - correct "Agency" reference

p2I31 - unit missing (cm-3?)

p2l32 - reference to Platnick and Twomey is missing

Table 1 - interquartile range: of the temporal variability of the domain-mean quantities? should be specified. Are LWP and Nd in-cloud or all-sky in both, observations and model results?

p3l14 - The quantities in Table 1 are not numerous, and don't include boundary layer properties

p4l20 - a reference for the Quickscat data source would be good

p5l10 - for completeness, the symbols $\tau, \mathrm{h}$, and $\mathrm{z}$ could be explained, too. $\rho_{w}$ presumably is a constant?

p6l6 - how do the authors come to the conclusion that these are "realistic"?

p7l6 - if the authors want to make this point, they should consider listing the observed range in Table 1.

Fig. 2 - how is cloud top defined? 
Table 2 - the caption should report the definition of "ship-seeded" / "ship-unseeded" as well as "detrained" and "wall"

p8 114 - the plume with $\mathrm{Na}>1000 \mathrm{~cm}-3$ seems to me much narrower than $60 \mathrm{~km}$. I would guess, only a few $\mathrm{km}$

p9|4 - were $\rightarrow$ was

Interactive

comment

p10 last line - reduction compared to what? to the ctrl simulation?

p12/7-9 - I have difficulties following this argument. What is meant by "domain-average Acld"? Is it what is labeled "albedo" in Table 2? It is hard to understand that the increase in in-cloud albedo by 5

p12/17 - this decrease in in-cloud LWP is not documented in the Tables or figures. Or is the area covered by detrained + wall the entire cloudy area?

p12l20 - given the relatively small mean increases in $\mathrm{Nd}$, and the fact that albedo is much less sensitive to $\mathrm{Nd}$ changes than to $\mathrm{L}$ changes, is this plausible? $\mathrm{Or}$ is the distribution of the relative changes of both quantities relevant?

p14l5 - it probably rather is "satellite-based estimates in CRE changes"

p14l16 - maybe specify "even in the absence"?

p16l1 - superfluous comma

p16l26 - the

p16l27 - "annual mean insolation"

p16l31 - extent

Supplement

Printer-friendly version

Fig. $2 \mathrm{a}$ - this seems to me an awkward definition of a CDF. I am used to CDFs that increase monotonically and asymptotically approach 1 (as in Fig. 5, in fact). 
Fig. $3 a-$ it would be good to indicate the ship position here, as well as the past ship track

Fig. 6 - this figure seems to be wrong. I believe the authors intended to show Nd_top vs. Acld histograms in all rows.

Interactive comment on Atmos. Chem. Phys. Discuss., https://doi.org/10.5194/acp-2018-708, 2018. 